\title{
University of Arizona's Collaboration to Advance Teaching Technology and Science (CATTS): lesson for photonics education collaborations
}

\section{Michelle Hall-Wallace, Nancy Regens, Stephen Pompea}

Michelle Hall-Wallace, Nancy L. Regens, Stephen M. Pompea, "University of Arizona's Collaboration to Advance Teaching Technology and Science (CATTS): lesson for photonics education collaborations," Proc. SPIE 4588, Seventh International Conference on Education and Training in Optics and Photonics, (28 May 2002); doi: 10.1117/12.468745

SPIE Event: Education and Training in Optics and Photonics 2001, 2001, Singapore, Singapore 


\title{
The University of Arizona's Collaboration to Advance Teaching Technology and Science (CATTS): Lesson for Photonics Education Collaborations
}

\author{
Michelle Hall-Wallace $^{\mathrm{a}}$, Nancy L. Regens ${ }^{\mathrm{a}}$, Stephen M. Pompea ${ }^{\mathrm{b}}$ \\ ${ }^{\text {a } C A T T S ~ P r o g r a m, ~ D e p a r t m e n t ~ o f ~ G e o s c i e n c e s, ~ U n i v e r s i t y ~ o f ~ A r i z o n a, ~} 1040$ E $4^{\text {th }}$ Street Tucson AZ \\ 85721-0077 USA ${ }^{1}$ \\ ${ }^{\mathrm{b}}$ Pompea \& Associates, 1321 East Tenth Street, Tucson AZ 85719-5808 USA ${ }^{2}$
}

\begin{abstract}
CATTS is a National Science Foundation-funded partnership between the University of Arizona and local school districts to improve science, mathematics and technology teaching at all levels. The goals of the CATTS Program are to develop sustainable partnerships with Kindergarten through 12th grade level (K-12) educators that foster integration of science, mathematics, engineering and technology research in classroom learning experiences. The program also creates opportunities for graduate and undergraduate students to be active participants in K-12 education by providing training and fellowships. CATTS seeks to foster effective teaching and a greater understanding of learning at all levels.
\end{abstract}

School districts and University of Arizona outreach programs propose fellowship activities that address identified educational needs; they work together with CATTS to create customized programs to meet those needs. CATTS Fellows, their faculty mentors and K-12 partners participate in workshops to gain experience with inquiry-based teaching and understanding diverse learning styles. In the partnership, CATTS Fellows have an opportunity to share their research experiences with K-12 educators and gain experience with inquiry teaching. On the other side of the partnership, professional educators share their knowledge of teaching with Fellows and gain deeper understanding of scientific inquiry. In the two years that this NSF funded program has been in operation, a variety of lessons have been learned that can apply to school, university, and industrial partnerships to foster education and training. In particular since each organization operates in its own subculture, particular attention must be paid to raising cultural awareness among the participants in ways that foster mutual respect and communication of shared goals. Proper coordination and sensible logistics are also critical for the success of a complex project such as this. Training of the partners and the project management will also be described.

Key words: professional development, education, training, GK-12, optics, photonics

\footnotetext{
${ }^{1}$ Dr. Michelle Hall-Wallace is a faculty member in the Department of Geosciences, College of Science at the University of Arizona. She is PI on the CATTS Project. She can be reached at hall@geo.arizona.edu. N. Regens is the Program Coordinator for the CATTS program and can be reached at nregens@geo.arizona.edu or http://www.geo.arizona.edu/catts/.

${ }^{2}$ Education Co-Chair, Arizona Optics Industry Association, Adjunct Faculty, University of Arizona, Telephone 520.792.2366 Email:spompea@pompea-associates.com
} 


\section{INTRODUCTION}

The field of optics/photonics is an inquiry-fueled field with many new applications developing each year. The challenge of developing a pipeline for highly skilled scientists and technicians for the optics and photonics industries is not unlike the challenges facing other scientific and high technology industries. However, the shortage of optics technicians and optical engineers is more severe due to the explosive growth of photonics in recent years and the breadth of skills needed for success in the field. For example, the main field of Information Technology and Telecommunications (information transport, information processing, optical storage, and displays) has advanced incredibly fast and hardly existed 20 years ago. Similarly, the areas of "Optics in the Health Care and Life Sciences and "Optical Sensing" have come into their own in recent years. ${ }^{1}$

Researchers and technicians in optics and photonics draw from physics, computer science, materials science and a range of engineering fields in their work. Developing an educational system or pipeline that draws attention to the career opportunities available and that assists K-16 faculty in preparing future photonics specialists is critical. One difficulty in attracting young people to scientific and technical careers is that often K-12 educators are prepared poorly for teaching mathematics and science and do not provide opportunities for students to experience the excitement and wonder of scientific discovery. Consequently, we lose many potential scientists and engineers before middle school. In this paper, we describe a partnership we developed to address the pipeline problem across the disciplines of mathematics, science and engineering. Our program is designed to make K-12 students aware of the tremendous career opportunities in these disciplines and provide K-16 faculty with resources and new skills to more effectively prepare students for these careers. Lessons learned from our experience can be easily applied to photonics and other fields.

Our program, the Collaboration to Advance Teaching Technology and Science (CATTS), provides highly qualified undergraduate and graduate students in the sciences, mathematics, engineering, and technology, their faculty mentors, and K-12 educators with opportunities to experience and practice inquiry-based teaching and to reflect on its implications for improved learning in these disciplines. Our goal is to develop a sustainable program that strengthens existing partnerships between the university faculty and students and K-12 educators in local school districts and leads to integration of research and education in science, mathematics, engineering and technology. These partnerships are central to the effective implementation of science education reforms ${ }^{2,3}$ needed to keep pace with our increasingly technological world and will hopefully provide future employees to growing industries like photonics and optics. With a grant from the NSF Graduate Teaching Fellowship program, CATTS is able to provide fellowships and associated training that enables the graduate and advanced undergraduate students (the Fellows) to serve as scientific and technical resources for K12 teachers and schools.

Expected outcomes of CATTS include improved communication and teaching skills for the Fellows, enriched learning experiences for K-12 students, professional development opportunities for K-12 teachers, and strengthened partnerships between the university and local school districts. In a survey of 1700 National Science Teachers Association members, 92\% of teachers who worked with scientists said it bolstered their motivation and enthusiasm for teaching science; $90 \%$ said it increased their understanding of the science content; and $87 \%$ said it improved their teaching ${ }^{4}$. Data 
like this bolsters our confidence that investing in a partnership with K-12 educators will result in students who have a greater interest in scientific and technical careers and who are better prepared to pursue those careers after high school.

\section{DEVELOPING THE PARTNERSHIP PLAN}

A partnership requires extensive coordination and communication among all interested parties: Fellows and their faculty mentors as well as K-12 educators and administrators ${ }^{5}$. In developing the concept for CATTS, we met with teachers, administrators and superintendents from local school districts to design a partnership that meets their needs. In addition, we involved graduate students and faculty conducting existing outreach efforts in discussions about how a CATTS partnership might enhance their programs. Our partnership plan incorporates the many ideas generated by these groups and reflects the community buy-in, which is an important part of our ongoing success. The partnership plan (1) identifies a range of partnership activities and the role of the Fellows; (2) defines the types of professional development that we will provide to the Fellows, mentors and K-12 partners; and (3) establishes a mechanism for the program coordinators to communicate, listen and respond effectively to the needs of all involved. We have continued a high level of communication with everyone involved in CATTS and try to maintain as much flexibility in the program to respond to individual needs. Communication has been the key to our success because it allows us to identify cultural and knowledge barriers early and then design training or other mechanisms to help the Fellows and partners over come them.

\section{A. Structure of the Partnership}

The CATTS program works on an 18-month cycle in response to the university and K-12 school schedules. In the fall semester, we solicit project ideas from local teachers, schools and school districts and university outreach programs. After identifying projects suitable for our program, we advertise them widely across the university campus. Many of our projects serve high minority schools thus, we specifically target under-represented groups and multi-lingual students in our recruitment. Interested students apply for a fellowship and indicate one or more projects that they would like to carryout. We select the Fellows by the end of the fall semester.

Recipients of the fellowship must complete a preparation program during the spring semester then, begin their fellowship and associated work in the schools during the following summer. Fellows must work 10 hours per week in the schools and 5 hours per week on preparation throughout the following year. Students receive their fellowship beginning in May after they have completed their training and the fellowship extends until May of the following year. Faculty mentors, especially those for the graduate students, provide guidance and encouragement to the Fellows. The climate of graduate school still focuses on research over teaching for graduate students thus, it is especially important to ensure the Fellow has his/her mentor s approval to participate. We work to increase that approval by incorporating the mentor in our training and social activities associated with the program. K-12 partners, whether district administrators or classroom teachers, are critical for providing opportunities for Fellows to explore the classroom when in training and to provide accurate information and insight into K-12 schools and students. The importance of the school connection in the training of the fellows cannot be overemphasized. 


\section{B. Program Activities and the Role of the Partners}

We have many University outreach programs, school district supported professional development programs and school based initiatives in which we can contribute and fulfill the goals of our program. We place 18-28 graduate or undergraduate Fellows each year in a variety of partnership roles. These activities and the corresponding role of the Fellows fall into three general categories, although there is much overlap between them. Fellows can serve as a:

(1) Resource agent implementing an existing university outreach program. In this model, outreach programs submit a proposal outlining activities that a Fellow will conduct for the program. For example, many of our outreach programs offer professional development workshops or graduate-level courses that introduce teachers to new curriculum materials or long-term, student-directed research projects supported by the program. A Fellow assigned to this type of project is directly involved in teaching the professional development workshops and provides the added bonus of helping teachers when they implement the new curriculum in their classrooms.

An example of this partnership is the Saguaro Project (http://saguaro.geo.arizona.edu). University scientists have developed an inquiry-based Earth science curriculum that uses a Geographic Information System. Prior to gaining assistance from the CATTS program only $25 \%$ of the teachers (representing 2 schools) who completed a threeweek Saguaro summer training workshop actually implemented the curriculum. With the assistance of a CATTS Fellow, 10 of the 22 high schools in our region have now implemented the curriculum in the past two years even though many of the teachers have only received 1-2 days of formal training. The Fellow works with school technology staff to install and test the software, assists the teacher with classroom implementation and even conducts one-day workshops for teachers to improve their skills using GIS.

(2) Resource agent directly connected with the professional development program of a local school district. This model is similar to the one above except that the district proposes and supervises the Fellows activities. Fellows work with master teachers to develop and conduct professional development workshops that train other classroom teachers who must implement newly adopted inquiry-based curriculum units. Fellows help teachers become comfortable with the inquiry process and the content in the unit while the master teacher provides teaching ideas and classroom management tips. A Fellow might also provide follow-up assistance by working directly with teachers to implement the curriculum in their classroom.

This model is in use in our largest school district, which is trying to implement a systemic change in elementary level science. The majority of teachers in elementary school are uncomfortable teaching science and mathematics. This combined with the emphasis on developing student s reading and writing in grades K-6 results in few teachers offering a high quality science learning experience for their students. The 
Fellows working on this project spend six to eight weeks in a school working with 3-4 teachers at one grade level. They assist them in all aspects of teaching these new inquiry modules. They also lead weekly workshops after school to help teachers become comfortable with the upcoming week s lessons. The district has observed that teachers working with the Fellows are more likely to implement the new curriculum and often have a change of attitude towards science. One teacher conveyed the following:

M. (the Fellow) helped me see the importance of science education. I have been so focused over the past 10 years on teaching reading and writing that everything else has been secondary.

$$
\text { Mrs. S- a second grade teacher. }
$$

Mrs. S was not the only one to change. The Fellow (M.) relayed the following about her experiences.

I came in early in the semester planning to measure Manduca larva with the students. Mrs. S pointed out that second graders don $t$ know how to measure with rulers. I was speechless for a moment, as I had not really thought of that. I recovered quickly and suggested that we use paper clips to measure and give children an idea of units. Instead, Mrs. S taught a small lesson on how to measure. She was able to anticipate what mistakes the children might make, like not starting at the end of the ruler, or starting at the wrong end of the ruler. The children picked it up and it amazed me! After that I realized that I had a great deal to learn about teaching and Mrs. S and I started planning almost every lesson together.

$$
M-a \text { CATTS Fellow }
$$

(3) CATTS Fellow in Residence at a local school. In this role a Fellow works with teachers in a school to enrich the science program at one or many grade levels. This model has been quite successful for scientists working with schools in England ${ }^{6}$. The school must work as a unit to identify ways that integrate the Fellow into their classrooms and curriculum before the Fellow arrives on campus. This process assures that both the Fellow and K-12 teachers have similar goals and expectations for the experience. In this model, a Fellow might help a teacher with a specific curriculum unit, provide professional development to advance the teacher s content knowledge in different areas, or work with small groups of students or the entire class conducting a focused research project. The Fellow could be assigned to one school for a year or rotate among several schools during the school year depending on the needs of the school and projects involved.

This is one of the most exciting and challenging assignments. The variety of activities a Fellow might engage in during the year is either large or very small. The model has been used to design and implement a long-term monitoring study for an endangered 
plant on one campus. It has also been used to develop an entirely new course in Earth science at a local high school. In this case, the Fellow worked closely with the teacher to gather new and innovative resources for the course during the summer months. During the school year, the teacher and Fellow share teaching responsibilities based on their individual expertise. Starting a new course is often overwhelming and fraught with miss-steps. In this case, the extra assistance of the Fellow has made it significantly more enjoyable and successful.

Most of our partnerships are focused on introducing new content materials (often related to new technology or scientific discoveries) into the classroom and helping teachers teach science more effectively. The instructional materials we implement must be aligned with the local and/or national education standards and be appropriate for the target audience to get buy-in from the teachers and schools. The optics and photonics industry could develop similar programs in communities where they have a strong presence and nurture the pipeline that will produce our future scientists and engineers. However, to be successful, these partnerships must reward both the teachers and the industry professionals willing to put forth the extra effort.

\section{Addressing Cultural Differences and Changing Cultural Norms}

A healthy and productive partnership depends on respect for and understanding of the needs, background and interests of all parties involved. Universities long ago recognized that partnerships with K-12 schools in the areas of K-12 for teacher preparation, recruiting a diverse student population, and stimulating interest in high demand careers were important. However, most universities are still reluctant to provide the faculty incentives and rewards that allow these partnerships to blossom. K-12 outreach is an integral part of our university s mission. Faculty are able to commit themselves to this mission because of a process for promotion and tenure which specifically rewards faculty engaged in K-16 science and mathematics education research and outreach $^{7}$. This new reward structure was critical to changing the culture at our university and creating opportunities for K-12 partnerships $^{8}$. Many faculty have redefined their roles and are more willing to allow their graduate students to engage in activities, such as outreach, that traditionally would be discouraged in favor of research. The CATTS program allows our graduate students to embrace and contribute to the university mission. Our hope is that this experience will help our graduate students institute cultural changes when they become faculty members at other institutions or work in industry.

While their intent is good, many university outreach programs have failed at building partnerships and effecting change in schools due to a lack of understanding of the K-12 culture and environment. The CATTS program fosters a two-way relationship in which university Fellows and faculty mentors can increase their understanding of issues in K-12 education while providing K-12 teachers the resources to improve their teaching of science of mathematics with inquiry. This understanding of cultures is developed in our preparation program for the Fellows, which also includes university faculty and K-12 teachers. The long-term outcome of CATTS is for the current and future professorate to become more effective inquiry-based teachers and in turn to prepare current and future K-12 teachers to create learning environments where inquiry and problem solving is the norm. If successful, we will have changed the cultures of both university and K-12 institutions. 


\section{Preparation of Fellows, Faculty Mentors and K-12 Educators}

A spring semester ( 2 credit / 30 hours) preparation workshop provides an environment in which the Fellows construct their understanding of the K-12 school environment, student competencies, the content-related needs of their teacher partners and effective methods for meeting some of those needs. Fellows learn how to be a resource to facilitate high quality instruction in the classroom. Through readings, discussions, focused classroom observations, guest speakers and inquiry-based activities, the Fellows develop an understanding of:

$¥$ their role in the schools;

$¥ \quad$ state and national science education standards ${ }^{2}$, mandatory testing and other programs;

$¥$ communicating sophisticated scientific ideas in simple terms;

$¥$ methods of inquiry-based instruction;

$¥$ the effective use of technology in teaching;

$¥$ methods for teaching diverse learners;

$¥$ questioning techniques and assessment strategies;

$¥$ sources of resource materials and other classroom support; and

$¥$ classroom management and general school district policies and procedures.

The basic format of the workshop is a series of topical sessions that build understanding of effective teaching methods in the first half of the semester followed by inquiry-based science investigations, classroom observations and working with K-12 teachers during the second half. Fellows learn to communicate scientific and mathematical concepts by applying the concepts taught in the topical sessions. They begin by teaching a single basic concept in a 2-minute presentation. Topics might include defining and providing a relevant application of a ratio, a semiconductor, a topographic map or a symbiotic relationship. They will also develop a short presentation or lesson on careers in their field to use as an introductory lesson. Teaching activities expand in length and depth over the semester. We encourage them to emphasize different teaching methods or skills in each experience. Surveys of the Fellow $s$ indicate that $75 \%$ begin the semester with

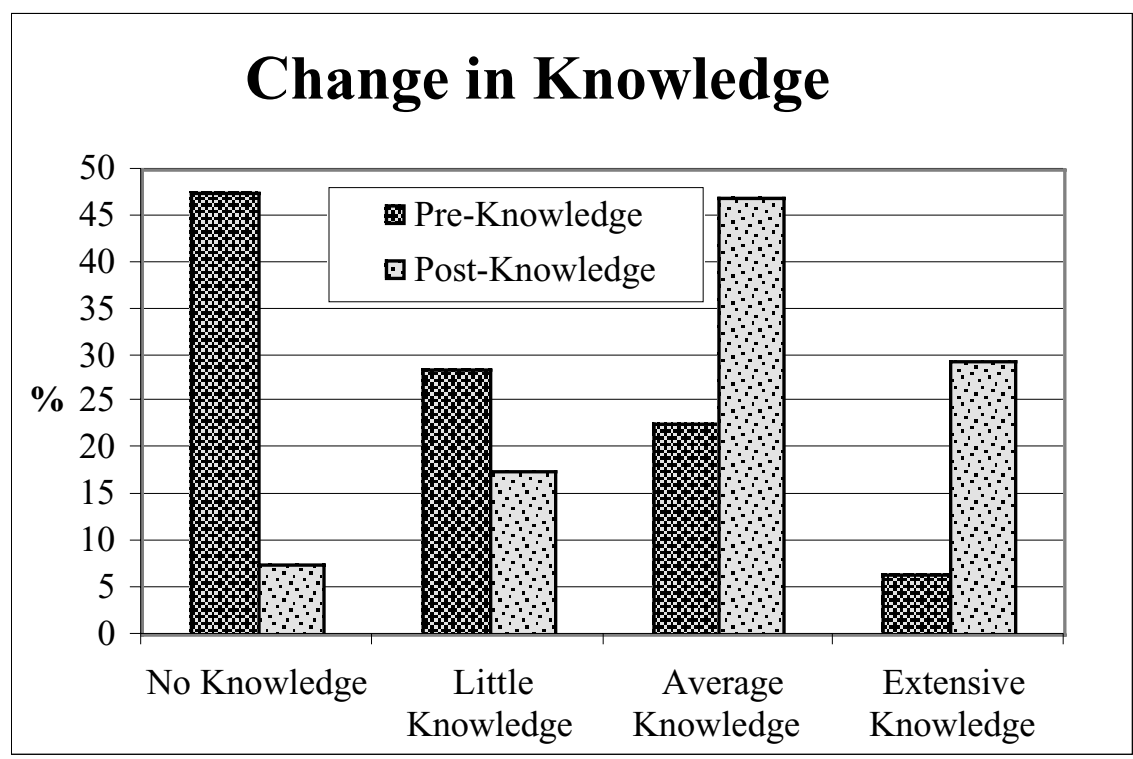

Figure 1: Fellows report a significant increase in knowledge about educational issues and concepts as a result of the preparation workshop. 
little knowledge or awareness of the core concepts and ideas in science education. By the end of the semester, $76 \%$ of the Fellow s reported that they had an average to exceptional knowledge of these concepts and ideas (Figure 1).

The inquiry-based activities used in the second half of the workshop are taken from the University $\mathrm{s}$ outreach programs or from local school districts mandated core curriculum. Along with experiencing inquiry first-hand, Fellows learn to evaluate curriculum for effectiveness in meeting state and national standards and ability to address learners with diverse learning styles. Interactions with K-12 teachers during the workshop and the classroom observations provide Fellows opportunities to enhance their partnerships. We rely on significant help from classroom teachers in conducting this workshop. Teachers conduct inquiry investigations, share their experiences through panel discussions and generally make themselves available to our students. The Fellows consistently rate the interactions with the teachers as the most valuable activity in helping them better understand the school/classroom community.

Faculty mentors and K-12 teachers working with the Fellows are encouraged to participate in three class sessions of their choice. The K-12 teachers receive professional development credit from their school district for their participation and an honorarium from the CATTS program. The professional development credit is important because it is required for salary increases. Faculty mentors have the opportunity to improve their own teaching by attending as well. The preparation workshop also offers an effective means for faculty mentors, Fellows and K-12 educators to become more knowledgeable about current outreach programs and to share expertise that could improve those programs.

During the fall semester after the Fellows begin working in schools, they attend a weekly one-credit (15 hours) seminar that helps them resolve obstacles to building a successful partnership. Fellows share experiences among themselves to improve their skills in outreach and education. This seminar provides critical support needed to keep the Fellows from feeling isolated in their outreach work. Discussions, presentations by teachers and readings are incorporated into the seminar to address particular needs that arise. During the second semester of the fellowship, the current Fellows are asked to occasionally participate in the preparation workshop for the following year s Fellows. The Fellows in the preparation workshop are encouraged to visit and observe the activities in classrooms where successful partnerships are in progress.

\section{PROJECT MANAGEMENT}

Project management is done on three levels. An advisory board consisting of the equal representation from the K-12 and university communities provides guidance to the Principal Investigator (Hall-Wallace) and the Program Coordinator (Regens) in the areas of recruitment and selection of projects and Fellows, budgets and community participation. The Principal Investigator oversees the budgets, assessment and provides more direct oversight of the project. She also teaches or co-teaches the workshop and seminar with another faculty member or the Program Coordinator.

The Program Coordinator is responsible for the majority of the day-to-day activities. This includes monitoring Fellows and assisting them with any problems, recruitment of projects and Fellows, 
communicating with school principals, classroom teachers and faculty mentors about the program, and many other tasks. To simplify supervision of the large number of Fellows, we require them to submit a weekly log that outlines how they completed their work for the previous week. We encourage the Fellows to also include any questions they have about their fellowship and to make requests for help if needed. These are very useful to us in heading off potential problems in the classrooms. The Fellows can be short sighted in this area due to their inexperience working in schools.

The Project Coordinator s role is to anticipate opportunities and obstacles that the Fellow may encounter and prepare them for events that might come up. When working in the schools, the Fellows are also required to submit a weekly schedule of their next week s work. The schedules allow the program coordinator to frequently visit them in the classroom without the need for prearrangements. This also gives Fellows an opportunity to invite us to attend some of the more fun and engaging activities taking place in their classrooms. To make it as easy for our Fellows to submit the information, we created interactive forms on our web site (http://www.geo.arizona.edu/catts/.

\section{B. Teacher Incentives}

Teachers have very busy lives and at first glance, working with a Fellow does not always seem like a benefit. Any disruption of a teacher s very full schedule, even when someone is trying to help can be stressful. Thus, communication with the teachers early and often is essential. When the partnerships take hold the direction of the projects can change depending on the interest of the teacher and Fellow. We provide $\$ 750$ to each Fellow to purchase supplies for a classroom teacher so that no matter which direction the partnership goes, a good idea will not be cast off due to lack of appropriate materials. Fellows have purchased anything from plants, shovels and gloves for a garden project to digital movies and a DVD player for a classroom. Most of the funds go to purchase of small items that allow the freedom to explore new directions in the curriculum. This feature is a real asset in building our partnerships.

In addition to the small grants for classroom materials and the opportunity for teachers to get professional development credit for working with the Fellows, we also include teachers in our informal social gatherings. Finally, we recognize the teachers with an attractive certificate signed by their Principal and school district Superintendent, the Dean of the College of Science at the University of Arizona and the Project Director.

\section{ASSESSMENT}

We have been evaluating our progress nearly continually in this project. To get direct feedback from the Fellows we asked them to keep a journal about their experiences. The journals are submitted monthly for comments and follow-up contacts are made with each Fellow as needed. These journals are critical to us in evaluating our program and in deciding what kinds of support services were needed for the Fellows. Through the journals we learned about our Fellows frustration at the beginning of the school year when they were not as successful as they expected in making change in

the classroom. Being youthfully idealistic, they expected change to come quickly and easily. We addressed these issues in our weekly seminar through open discussions. When the Fellows realized 
that they were all having similar troubles, it both relieved their anxiety and helped them become more creative and assertive in their approach to the schools. At the end of the fellowship these same fellows were happy with small, incremental changes, realizing that is how substantive, long-term change occurs. One of our Fellows called these small changes, Geek Victories . An example of a Geek Victory was when her $7^{\text {th }}$ grade math students could trouble shoot problems when trying to enter a formula in a spreadsheet. Another occurred when students realized they could not divide by zero.

Student journal writing also revealed the need for Fellows to work in the schools more during their spring training workshop. Although our Fellows had told us that our spring workshop had prepared them well for their school experiences, we felt that more time in K-12 classrooms would make it easier to get teachers engaged with the Fellows in the fall. Our exit interviews reinforced our conclusion that the Fellows need to start their collaborations with K-12 teachers before the busy early fall period. With this knowledge we increased the amount of K-12 classroom visits required for the second group of Fellows during their pre-fellowship workshop. As a consequence, this second group felt they were better prepared to work with the K-12 culture than the first group did. An added benefit of these increased visits was that the new cohort of Fellows was able to work in the same classroom as the first year Fellows. The first year Fellows were tremendously supportive of the new Fellows and helped them build relationships with the teachers.

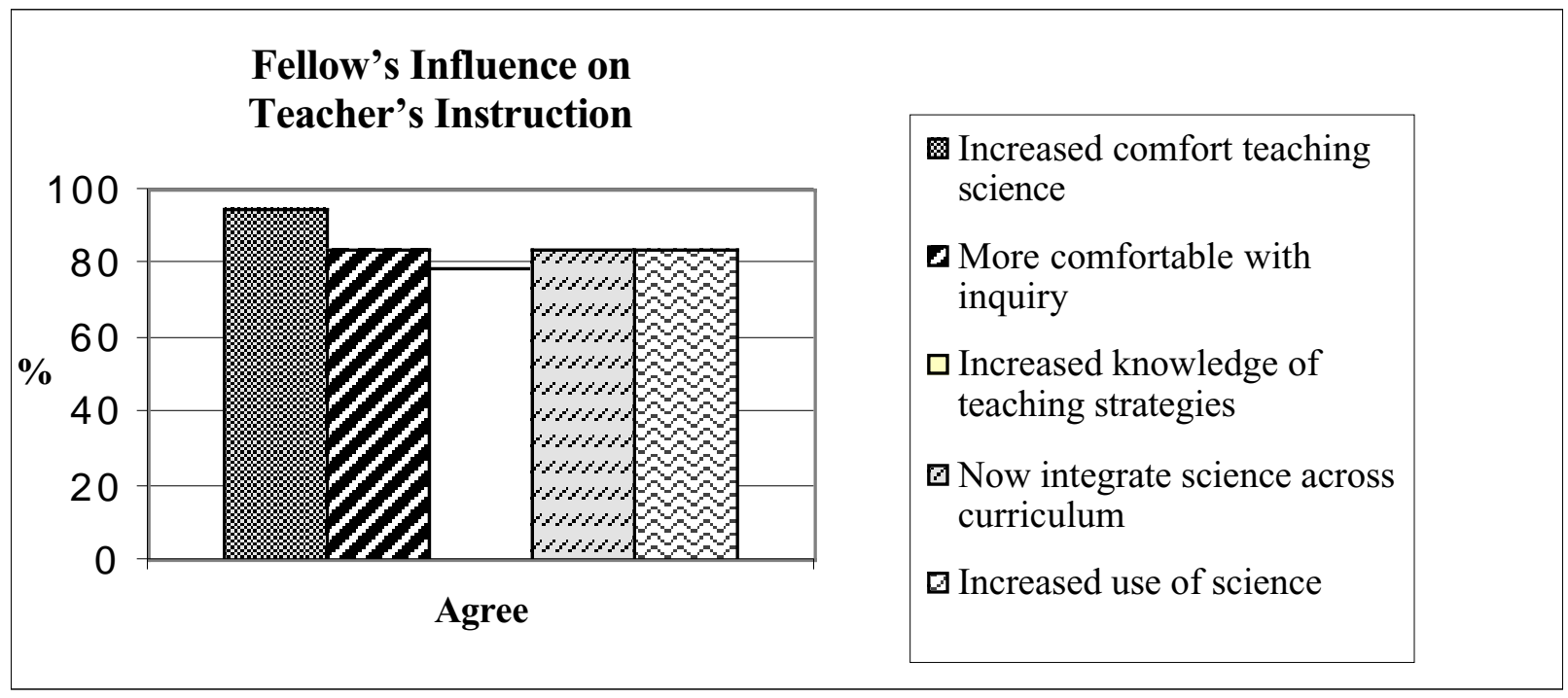

Figure 2: Teachers report more comfort teaching science and using inquiry in the classroom as a result of working with a Fellow.

Three times during the first cycle of the fellowship, we asked Fellows to complete surveys of their knowledge (see Figure 1) and attitudes about K-12 science education. At the end of the fellowship, we surveyed our Fellows and conducted a tape-recorded interview. Each fellow also submitted a short end of the fellowship project status report. Finally, participating K-12 teachers were also surveyed about how they felt the partnership had proceeded and how we could improve our program. Two key results from the teacher survey suggest that we are having a lasting impact in the classroom. Figure 2 shows responses of teachers about changes in the use of inquiry and the amount 
of science they now teach in their classroom. In both categories, we see very positive changes due to the efforts of the Fellows. This suggests that the one-year partnership will have an impact on many students in the future. Due to the large volume of data, the full results of these assessments cannot be presented here. However, each of these assessments provided ideas for small adjustments that have continually improved our program.

\section{CONCLUSION}

The gap is growing between the number of scientists and engineers needed to fuel the demands of our new global economy and high technology society and the number available. This is especially true in rapidly emerging fields such as photonics and optics. Current educators in our public schools and even in some colleges and universities are not well equipped to prepare future generations of students for careers in these fields. To meet the challenge will require partnerships between the industry, K-12 schools, colleges and universities. These partnerships must acknowledge and respond to differences in cultures and responsibilities among all participants. They must also provide incentives and rewards for industry scientists and engineers, as well as educators in K-16, to participate.

\section{ACKNOWLEDGEMENTS}

The University of Arizona's Collaboration to Advance Teaching Technology and Science (CATTS) program is sponsored under grant 9979670 from the National Science Foundation.

\section{REFERENCES}

${ }^{1}$ National Research Council, Harnessing Light-Optical Science and Engineering for the 21st Century, National Academy Press, 1998.

2 National Research Council, 1996, National Science Education Standards, National Academy Press, Washington, D.C.

${ }^{3}$ F. J. Rutherford and A. Ahlgren, Science for All Americans, Oxford University Press, pp. 272 , 1990.

${ }^{4}$ Management Recruiters International, Weekly Bulletin, April 19, 1999.

5 A. Sussman, Science Education Partnerships: Manual for Scientists and K-12 Teachers, Ed. Art Sussman, University of California, San Francisco, 1993.

${ }^{6}$ S. Natynczuk, Confessions of a live-in scientist, New Scientist, Nov. 30, pp. 61-62, 1991.

${ }^{7}$ J. Alper, Scientists return to the elementary school classroom, Science, pp. 68-69, 1994.

${ }^{8}$ M. Hall-Wallace, How education became one university s priority, Geotimes, v. 45, n. 9, pp. $14-$ $16,2000$. 